\title{
BMJ Open Ghost ileostomy versus conventional loop ileostomy in patients undergoing low anterior resection for rectal cancer (DRKS00013997): protocol for a randomised controlled trial
}

\author{
Felix J Hüttner (D) , ${ }^{1,2}$ Pascal Probst (1) , , ${ }^{1,2}$ André Mihaljevic, ${ }^{1}$ Pietro Contin, ${ }^{1}$ \\ Colette Dörr-Harim, ${ }^{1,2}$ Alexis Ulrich, ${ }^{3}$ Martin Schneider, ${ }^{1}$ Markus W Büchler, ${ }^{1}$ \\ Markus K Diener, ${ }^{1,2}$ Phillip Knebel ${ }^{1}$
}

To cite: Hüttner FJ, Probst P, Mihaljevic A, et al. Ghost ileostomy versus conventional loop ileostomy in patients undergoing low anterior resection for rectal cancer (DRKS00013997): protocol for a randomised controlled trial. BMJ Open 2020;10:e038930. doi:10.1136/ bmjopen-2020-038930

- Prepublication history and additional material for this paper are available online. To view these files, please visit the journal online (http://dx.doi. org/10.1136/bmjopen-2020038930).

Received 01 April 2020 Revised 26 August 2020 Accepted 25 September 2020

D) Check for updates

(c) Author(s) (or their employer(s)) 2020. Re-use permitted under CC BY-NC. No commercial re-use. See rights and permissions. Published by BMJ.

For numbered affiliations see end of article.

\section{Correspondence to}

Prof. Dr. Markus W Büchler; markus.buechler@med.uniheidelberg.de

\section{ABSTRACT}

Introduction Anastomotic leakage is the most important complication in colorectal surgery occurring in up to $20 \%$ after low anterior rectal resection. Therefore, a diverting ileostomy is usually created during low anterior resection to protect the anastomosis or rather to diminish the consequences in case of anastomotic leakage. The socalled virtual or ghost ileostomy is a pre-stage ostomy that can be easily exteriorised, if anastomotic leakage is suspected, in order to avoid the severe consequences of anastomotic leakage. On the other hand, an actual ileostomy can be avoided in patients, who do not develop anastomotic leakage.

Methods and analysis The GHOST trial is a randomised controlled pilot trial comparing ghost ileostomy with conventional loop ileostomy in patients undergoing low anterior resection with total mesorectal excision for rectal cancer. After screening for eligibility and obtaining informed consent, a total of 60 adult patients are included in the trial. Patients are intraoperatively randomised to the trial groups in a 1:1 ratio after assuring that none of the intraoperative exclusion criteria are present. The main outcome parameter is the comprehensive complication index as a measure of safety. Further outcomes include specific complications, stoma-related complications, complications of ileostomy closure, frequency of transformation of ghost ileostomy into conventional ileostomy, frequency of terminal ostomy creation, proportion of patients with an ostomy at 6 months after index surgery, anorectal function (Wexner score) and quality of life assessed by the European Organisation for Research and Treatment of Cancer (EORTC) QLQ-C30 and CR29 questionnaires. Follow-up for each individual patient will be 6 months.

Ethics and dissemination The GHOST trial has been approved by the Medical Ethics Committee of Heidelberg University (reference number S-694/2017). If the intervention proves to be safe, loop ileostomy could be spared in a large proportion of patients, thus also avoiding stoma-related complications and a second operation (ileostomy closure) with its inherent complications in these patients.
Strengths and limitations of this study

- This is the first randomised controlled pilot trial comparing the so-called ghost ileostomy to the current standard of care, protective loop ileostomy, in patients undergoing low anterior resection with total mesorectal excision for rectal cancer.

- Strengths of this study are the randomised design and the application of valid applicable definitions for the main endpoints.

- If the intervention proves to be safe, a loop ileostomy could be spared in a large proportion of patients, thus also avoiding stoma-related complications and a second operation (ileostomy closure) with its inherent complications in these patients.

- A limitation is that this trial is a pilot trial with limited sample size to acquire preliminary data, which will form the basis for a subsequent larger confirmatory multicentre trial.

Trial registration number German Clinical Trials Registry (DRKS00013997); Universal Trial Number: U1111-12089742.

\section{INTRODUCTION}

Colorectal cancer still ranks under the top three cancer sites and cancer-related deaths worldwide. Approximately $30 \%-40 \%$ of all colorectal carcinomas are located in the rectum requiring specific treatment strategies due to its anatomical and physiological features. ${ }^{1}$ The mainstay of modern, multimodal rectal cancer treatment remains oncological resection, performed as a sphincter-sparing procedure in terms of low anterior resection with total mesorectal excision (LAR/TME) whenever possible. However, this operation bears the risk of anastomotic leakage (AL), a potentially 


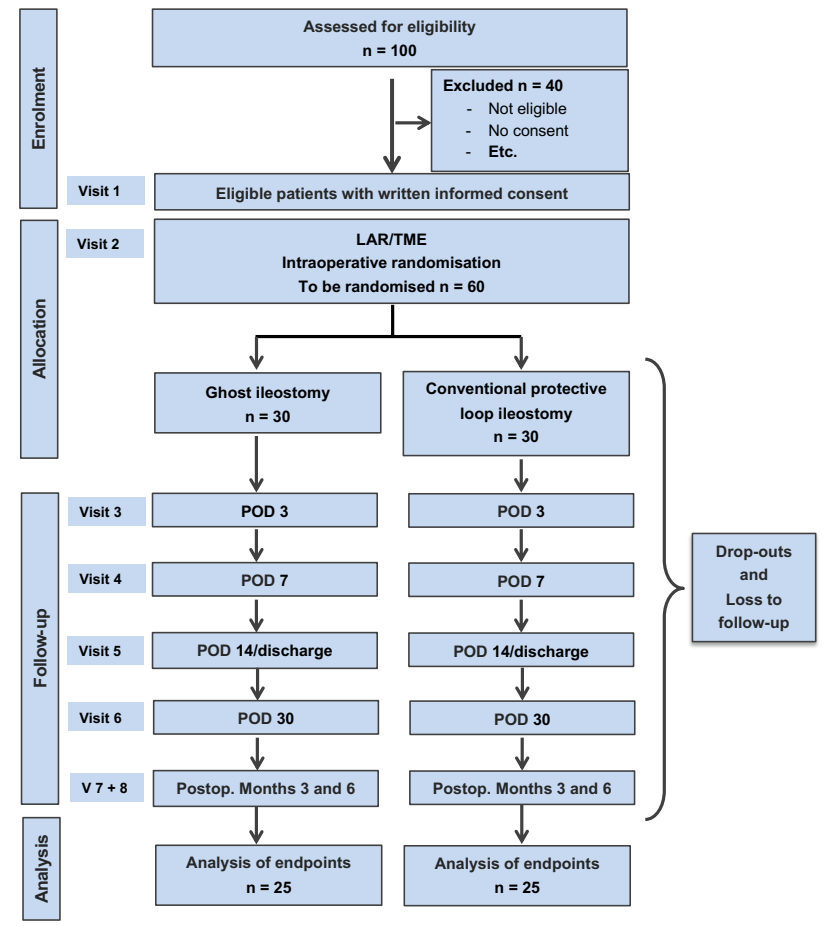

Figure 1 Trial flow chart. LAR/TME, low anterior resection with total mesorectal excision; POD, postoperative day.

life-threatening complication, with a reported frequency ranging from $3 \%$ to $23 \%$ in the scientific literature. ${ }^{2}$ Therefore, most surgeons prefer and some guidelines even demand the construction of a diverting stoma to prevent AL or rather diminish its consequences. ${ }^{3-5}$ On the other hand, stoma-related complications such as peristomal abscess, parastomal hernia or dehydration due to high-output stoma culminating in acute kidney injury, and the need for a second operation, that is, stoma reversal, have to be considered in the decision for routine use of a diverting ileostomy in all patients undergoing LAR/ TME ${ }^{67}$ Furthermore, an ostomy may impact quality of life (QoL) and long-term anorectal function. ${ }^{8}$ Assuming a frequency of AL of approximately $20 \%$ means that four out of five patients receive a loop ileostomy without a compelling need or even unnecessarily. Therefore, strategies for a more selective practice regarding the creation of diverting ileostomies in these patients are urgently wanted.

In 2007, Sacchi et al described their technique of a 'virtual ileostomy' for the first time, representing a prestage ileostomy that can be opened by a short surgical procedure, if any signs of AL are present. ${ }^{9}$ As a consequence of this approach, a large proportion of patients will not receive a 'real' stoma, whereas in patients developing AL, early transformation of the 'virtual' or ghost ileostomy into a loop ileostomy is deemed able to diminish the clinical consequences of AL comparable to a routine protective ileostomy. Since its first introduction, several authors have adopted the technique of ghost ileostomy and reported their respective results. ${ }^{10-14}$ However, most of these previous reports were retrospective case series or case-control studies despite two prospective controlled studies and one randomised controlled trial (RCT) that compared ghost ileostomy with a no-stoma group. ${ }^{15-17} \mathrm{~A}$ recent systematic review summarising the results of all previous studies regarding the ghost ileostomy concluded that it is a safe and effective alternative to routine ileostomy with a rate of exteriorisation of the ghost ileostomy of $10.5 \%$ and a rate of adverse events related to the ghost ileostomy of $2.1 \% .{ }^{18}$ However, all previous studies exhibited substantial risk of bias, in particular selection bias. Furthermore, the only RCT is not generalisable to the clinical situation in most countries since in the control group no stoma at all was created limiting its external validity. ${ }^{17}$ Therefore, the results of these studies are promising, yet the resulting evidence is not sufficient to justify its routine use.

The aim of the GHOST trial is to assess whether ghost ileostomy is a safe alternative to the current reference standard of conventional loop ileostomy in patients undergoing elective LAR/TME for rectal cancer. This strategy could potentially spare a loop ileostomy for a large proportion of patients and also avoid stoma-related complications and the need for a second operation, that is, ileostomy closure, with its inherent complications. Furthermore, the acquired data from this trial will form the basis for a subsequent larger confirmatory trial, if ghost ileostomy proves to be a safe alternative to conventional ileostomy.

\section{METHODS AND ANALYSIS \\ Trial design}

The GHOST trial is a randomised controlled pilot trial at the development stage (according to the IDEAL recommendations) ${ }^{19} 20$ with two parallel trial groups and a 1:1 allocation ratio. Initially the trial was planned as a monocentre trial, but because of slow recruitment, the trial is currently expanded into a multicentre design with two to three further centres that are presently initiated.

Patients scheduled for elective low anterior resection for rectal cancer at the trial centres will be assessed for eligibility and will be informed about the trial in detail. After providing written informed consent (for a model consent form see online supplemental file 1), a total of 60 eligible patients will be randomised in a 1:1 ratio within the trial. Assuming a dropout rate of 10 patients during trial duration, data of 25 patients in each group will be finally analysed (figure 1). This number was estimated to be sufficient for a preliminary assessment of safety of the trial intervention. The results of the current exploratory pilot trial will serve as basis for sample size calculation of a subsequent confirmatory multicentre RCT. 


\section{Box 1 Inclusion and exclusion criteria of the trial}

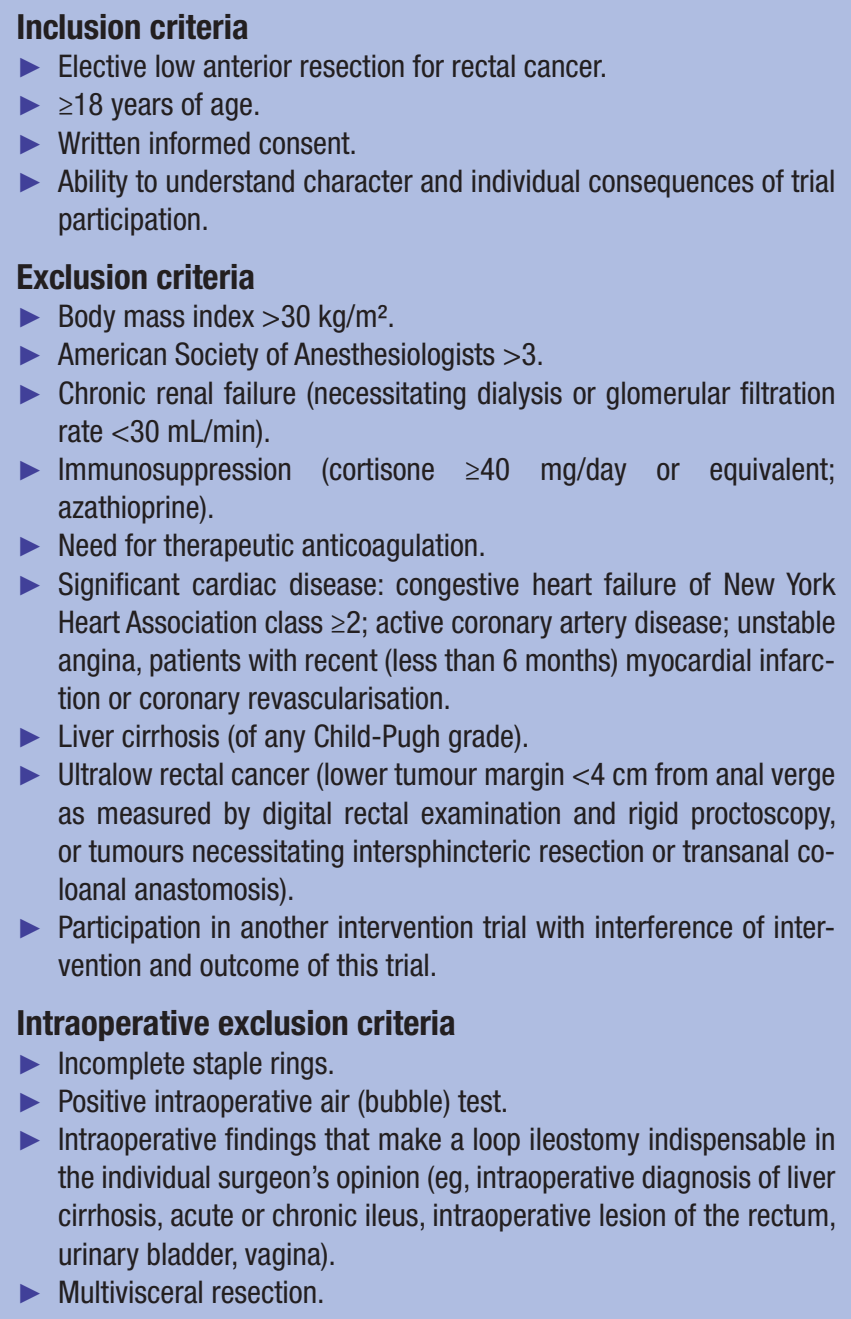

\section{Trial population and eligibility criteria}

Adult patients undergoing low anterior resection with total mesorectal excision for rectal cancer at one of the trial centres will be included. The following preoperative and intraoperative exclusion criteria were chosen in order to exclude patients at high risk of anastomotic leakage (box 1 ).

\section{Subject withdrawal}

Patients are free to leave the trial at any time and without giving reasons for their decision. Subjects may be withdrawn from the trial for the following reasons:

1. Patients may decide to withdraw from the trial at any time without providing any specific reason for their decision.

2. If, in the investigator's opinion, continuation of the trial would be detrimental to the subject's well-being. In case of (2), the reason for withdrawal must be recorded in the case report form (CRF) and in the patient's medical records.

\section{Sample size}

GHOST is a pilot trial focusing on safety and feasibility of the procedure; therefore, no formal sample size calculation has been performed. The total of 30 randomised and at least 25 analysable patients in each group were judged to be sufficient for assessment of safety and a preliminary sample size calculation for a subsequent larger confirmatory trial. This is in accordance with the suggested rule of thumb for sample size estimation in pilot trials by Whitehead $e t a l$, if a small standardised effect size $(0.1 \leq \delta \leq 0.3)$ is expected and the main trial's sample size will be calculated with a power of $90 \%{ }^{21}$

\section{Trial-specific procedures}

Standard perioperative management in both groups

Patients in both groups will undergo mechanical bowel preparation by means of polyethylene glycol solution combined with oral antibiotics (metronidazol $400 \mathrm{mg}$ orally two times per day+paromomycin $8000 \mathrm{mg}$ orally once) on the day before surgery according to local standards. Preoperatively, a designated ileostomy location will be marked at an appropriate place by one of the treating physicians or a stoma nurse usually at a position inside the rectus sheath.

Postoperative care will include early mobilisation and early oral intake starting with liquids 4 hours after surgery and resumption of an oral diet on the day after surgery. Postoperative clinical management will consist of daily ward rounds with clinical assessment by surgeons and routine blood tests usually performed on postoperative days $1,3,5$ and thereafter on demand based on the clinical course. Further diagnostic measures (eg, CT scans, proctoscopy) will be performed as necessary based on the clinical course of the patients and the judgement of treating physicians.

\section{Standard surgical technique performed in both groups}

Access to the abdomen can either be achieved by median laparotomy or by laparoscopy. LAR/TME and reconstruction of the bowel continuity by descendo-rectostomy will be performed according to local standards. Briefly, the left-sided colon will be mobilised up to the middle colic artery. The inferior mesenteric vein will be divided at the inferior border of the pancreas and subsequently, the inferior mesenteric artery will be divided close to its origin from the aorta. After routine total mesorectal excision with sphincter preservation, a resection of convenience at an appropriate location on the descending colon will be performed. Perfusion of the distal end of the descending colon will be assessed by direct inspection of a pulsatile bleeding and palpation or visible pulsating perfusion. For reservoir reconstruction, either a transverse coloplasty with end-to-end anastomosis or a side-to-end descendorectostomy will be performed. A double-stapling anastomosis will be fashioned by transanal circular stapling of the appropriate size $(28,29,31$ or $33 \mathrm{~mm})$. The spike of the transanally introduced stapler should perforate the rectal stump just below or just above the linear stapling line in a central position. After creation of the anastomosis, its integrity will be tested by transanal air insufflation. For this purpose, the proximal colon will be 
A)

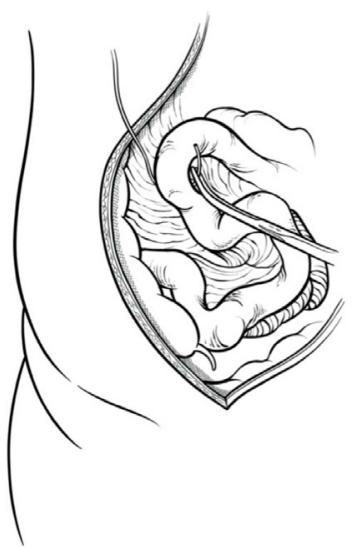

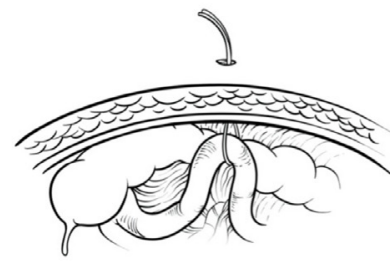

Figure 2 Schematic illustration of the ghost ileostomy (A) silicone loop is passed around the terminal ileum; (B) silicone loop is exteriorised, while the terminal ileum remains intraabdominal without tension.

compressed manually in open surgery or by atraumatic forceps in minimally invasive cases. Thereafter, a blind syringe with air will be used to insufflate the rectum transanally. Only if no technical problems during the procedure and no signs of leakage are present, the patient will be randomised to either ghost ileostomy or conventional loop ileostomy.

\section{Experimental intervention (ghost ileostomy)}

After construction and air testing of the rectal anastomosis, the last loop of the small bowel, that is, terminal

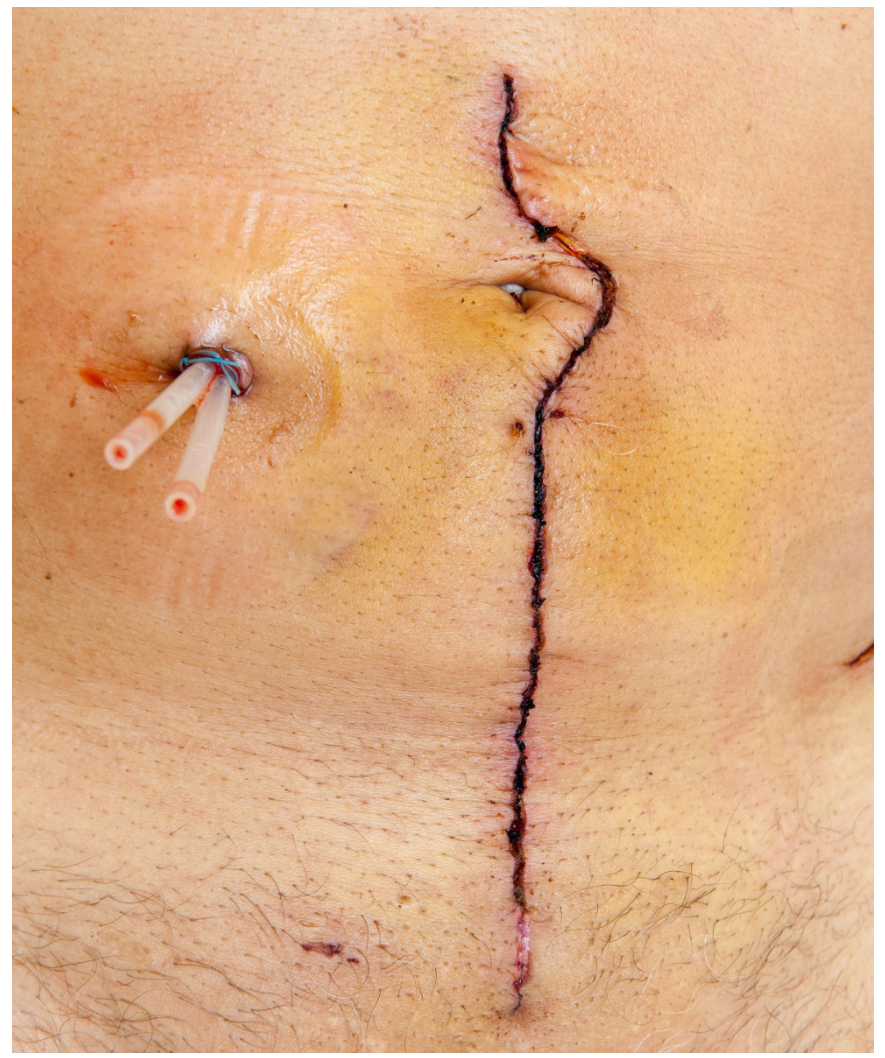

Figure 3 Photograph of a patient with ghost ileostomy prior to removal of the silicone loop on postoperative day 6 .

ileum, will be identified. A mesenteric window will be prepared at the designated ghost ileostomy site and a silicone loop will be passed through the mesenteric window around the small bowel. To assure correct orientation of the bowel in case that the ghost ileostomy has to be exteriorised, the oral part of the ileum will be marked about 1-2 $\mathrm{cm}$ proximally to the silicone loop with a serosal, non-absorbing suture. At the preoperatively marked, designated ileostomy location, the silicone loop will be exteriorised by a stab incision and fixed on the skin with a rod and non-absorbing sutures so that the bowel remains below the abdominal fascia without any tension. Thereafter, the abdomen will be closed in standard manner and the midline incision or laparoscopy incisions will be sutured and all incisions as well as the exteriorised silicone loop will be draped with sterile dressings. A schematic illustration of the ghost ileostomy is displayed in figure 2 and a photograph of a patient with ghost ileostomy is displayed in figure 3 .

Patients will be closely monitored for any signs of anastomotic leakage. Clinical and laboratory signs consist but are not limited to the following: fever, abdominal guarding, peritonism, air/faecal/turbid discharge from the intra-abdominal drains, leucocytosis or elevated $\mathrm{C}$ reactive protein values representing a deviation from the expectable postoperative course, any radiological or palpatory evidence of anastomotic leakage. If anastomotic leakage is suspected in a patient with ghost ileostomy, the ghost ileostomy will be exteriorised in the operating room under general anaesthesia. In the same manner as for conventional ileostomy, a longitudinal cylinder of skin and subcutaneous tissue will be excised alongside the silicone loop. The ventral and dorsal sheath of the abdominal fascia will be incised similarly as for conventional ileostomy and the terminal ileum will be exteriorised by gentle traction on the silicone loop. The proximal segment of the small bowel will be identified by the serosal suture that has been placed during primary surgery and the stoma will be created in correct orientation by circular everting sutures in standard manner, that is, without torsion on the mesentery or bowel with the oral ileal limb placed caudally if possible. Finally, an ileostomy bag will be placed.

Further diagnostic (proctoscopy, CT scan) or therapeutic measures (interventional drainage) will be performed in all patients with suspected anastomotic leakage depending on the clinical course and according to the routine clinical decisions of the treating physicians.

If no signs of anastomotic leakage occur after ghost ileostomy, the patient has regular bowel movements and is fed completely per os, the silicone loop will be removed at the bedside on postoperative days 5-10.

\section{Control intervention (loop ileostomy)}

After construction and air testing of the anastomosis, the last loop of the small bowel, that is, terminal ileum, will be identified in the same manner as in the experimental group. A mesenteric window will be prepared at 
the designated ileostomy loop and a silicone loop will be passed through the mesenteric window around the small bowel. For conventional ileostomy, a longitudinal cylinder of skin and subcutaneous tissue will be excised. The ventral and dorsal sheath of the abdominal fascia will be incised in a way that two fingers pass easily through the opening. Then, the ileal loop will be exteriorised. The stoma will be created in correct orientation by circular everting sutures in standard manner, that is, without torsion on the mesentery or bowel with the oral ileal limb placed caudally if possible. Finally, an ileostomy bag will be placed.

\section{Ileostomy closure (both groups)}

Ileostomy closure will be usually performed at the earliest 12 weeks after primary surgery or after termination of adjuvant therapy. Before closure of the ileostomy, a watersoluble contrast enema will be performed in all patients to exclude radiological or persisting anastomotic leakage. Further preoperative diagnostics, for example, proctoscopy or sphincter manometry will be performed at the discretion of the treating physicians. Ileostomy closure will be performed in standard manner in both groups, if there were no contraindications during the preoperative work-up.

\section{Permitted/prohibited treatments and concomitant treatment}

Any concomitant or necessary treatment, for example, antibiotic therapy, parenteral nutrition and relaparotomy will be performed at the discretion of the treating physicians and according to local standards.

\section{Data capture and trial endpoints}

The following baseline and demographic parameters will be captured in all patients: age (years), gender (female/ male), height $(\mathrm{cm})$, weight $(\mathrm{kg})$, previous abdominal surgery, American Society of Anesthesiologists class, histologically proven carcinoma (yes/no), bowel obstruction (yes/no), rectal bleeding/anaemia (yes/no), height of the tumour from anal verge $(\mathrm{cm})$, neoadjuvant therapy (yes/no), relevant comorbidities/medical history (cardiac, pulmonary, renal, hepatic, diabetes) and prior imaging studies (CT/MRI/endoscopic ultrasound).

Intraoperatively, despite checking the intraoperative exclusion criteria, the following data will be gathered: duration of surgery (min), surgeon's expertise ( $>$ or $\leq 50$ low anterior resections previously), intraoperative blood loss $(\mathrm{mL}$ ), intraoperative blood transfusion (yes/no and number of packed red blood cells), reconstruction technique, minimally invasive/open surgery, height of anastomosis $(\mathrm{cm})$ and drainage (yes/no).

The main outcome parameter of the trial will be the Comprehensive Complication Index (CCI). The CCI summarises all postoperative complications based on the established Clavien-Dindo classification (ranging from mild complications not leading to a deviation from the normal clinical course (grade I) up to postoperative death (grade V)) at an individual patient level according to their grade of severity. ${ }^{22}$ The CCI will be calculated for the period of initial hospital stay, for 3 and 6 months postoperatively.

Further outcome parameters are listed in the following:

1. Postoperative complications graded according to the Clavien-Dindo classification. ${ }^{23}$

2. Anastomotic leakage (according to the International Study Group of Rectal Cancer definition; including grading). ${ }^{2}$

3. Frequency of transformation of the ghost ileostomy into a protective loop ileostomy.

4. 30-day mortality.

5. Relaparotomies.

6. Terminal ostomy (Hartmann's procedure or for example, abdominoperineal extirpation).

7. Intra-abdominal abscess, defined as intra-abdominal collection of purulent or infected fluid (positive microbiological culture) confirmed by typical radiological findings (eg, CT scan), interventional drainage or during surgical reintervention.

8. Interventional drainage.

9. Completion of intended perioperative or adjuvant chemotherapy.

10. Patients with stoma (terminal/loop) at 6 months after initial surgery.

11. Stoma-related complications.

- Peristomal wound/abscess/ulceration, defined as erythema and/or dermal excoriation and/or induration and/or putrid collection in direct proximity to the ileostomy leading to pain, burning and difficulties with placement of the ileostomy bag.

- Parastomal hernia.

- Dehydration/high output with the need for intravenous-fluid substitution, defined as ileostomy output of $>1500 \mathrm{~mL}$ per day together with clinical signs of dehydration and/or reduced kidney function.

- Acute kidney injury according to KDIGO definition. $^{24}$

- Other stoma-related complications.

12. Complications after ileostomy closure.

- Anastomotic leakage.

- Prolonged postoperative ileus, defined as presence of two or more of the following criteria:

- Nausea/vomiting.

- Inability to tolerate oral diet over the last 24 hours.

- Absence of flatus or stool over the last 24 hours.

- Abdominal distension.

- Radiological confirmation.

- Wound dehiscence.

- Surgical site infection (Centers for Disease Control and Prevention). ${ }^{25}$

- Burst abdomen (dehiscence of abdominal fascia).

- Incisional hernia.

- Reoperation.

13. Mean length of bearing a stoma (in days). 
14. Overall postoperative length of hospital stay (primary and all subsequent hospitalisations).

15. Postoperative anorectal function 6 months after initial surgery (Wexner score) ${ }^{26}$

16. QoL (European Organisation for Research and Treatment of Cancer QLQ-C30, CR29) at baseline, 3 and 6 months after surgery. ${ }^{27} 28$

17. Survival.

\section{Patient timelines and description of trial visits}

Patients scheduled for elective LAR/TME will be screened during outpatient consultation or on the day of hospital admission. After a patient has given written informed consent, and all eligibility criteria have been checked during the screening visit, the above-mentioned baseline data will be assessed and documented (visit 1).

Randomisation will be performed during surgery after checking the intraoperative eligibility criteria by authorised personnel (visit 2). During this visit, the abovementioned intraoperative parameters will be assessed. Patients are followed up for 6 months after LAR/TME with follow-up visits scheduled on postoperative days 3 and 7 (visit 3 and 4), 14/day of discharge (visit 5), as well as 1 (visit 6), 3 (visit 7) and 6 (visit 8) months postoperatively. Visits 6-8 will be performed either during outpatient follow-up consultations or as telephone visits. During postoperative visits primary and secondary endpoints are assessed and documented. Furthermore, diagnostic and therapeutic interventions/operations due to postoperative complications will be assessed and documented. Table 1 gives an overview about trial visits.

\section{Safety objectives and assessment of safety}

Safety will be assessed by means of the CCI, mortality and the specific complications mentioned above. Since this is not a drug trial, no classical assessment of (serious) adverse events is mandatory. In fact, adverse events are represented by complications of grades 1-2 according to the Clavien-Dindo classification, whereas grades 3-5 represent serious adverse events. ${ }^{23}$ For all complications it will be assessed, whether the complication was related to the index surgery (LAR/TME), or if it was a stomarelated complication, or a complication related to ileostomy closure.

\section{Data handling and monitoring}

Data capture and management will be performed using REDCap electronic data capture tool hosted at the coordinating investigator's institution. ${ }^{29}$ All protocol-required information collected during the trial will be entered by the investigator, or a designated representative, in the electronic CRF. The investigator, or designated representative, should complete the CRF as soon as possible after information is collected, preferably on the same day that a trial subject is seen for an examination, treatment or any other trial procedure. Any outstanding entries must be completed immediately after the final examination. An explanation should be given for all missing data.

The completed CRF will be reviewed and signed by the investigator or by a designated subinvestigator.

The completeness, validity and plausibility of data will be examined by validating programmes, which thereby generate queries. The investigator or the designated

Table 1 Trial visits and documented parameters

\begin{tabular}{|c|c|c|c|c|c|c|}
\hline \multirow[b]{3}{*}{ Visit } & V1 & V2 & V3 & V4 & V5 & V6-V8 \\
\hline & $\begin{array}{l}1 \text { week to } 1 \text { day } \\
\text { before surgery } \\
(-7 \text { to }-1)\end{array}$ & $\begin{array}{l}\text { Day of surgery } \\
\text { (day 0) }\end{array}$ & POD 3 & POD 7 & $\begin{array}{l}\text { POD 14/ } \\
\text { discharge }\end{array}$ & $\begin{array}{l}\text { 1/3/6 months, postop/ } \\
\text { EoS, premature trial } \\
\text { termination }\end{array}$ \\
\hline & \multicolumn{5}{|c|}{ Inpatient } & Outpatient/telephone \\
\hline Informed consent & $\mathbf{x}$ & & & & & \\
\hline $\begin{array}{l}\text { Demographics and } \\
\text { baseline clinical data }\end{array}$ & $\mathbf{x}$ & & & & & \\
\hline $\begin{array}{l}\text { Eligibility criteria } \\
\text { (intraoperative) }\end{array}$ & & $\mathbf{x}$ & & & & \\
\hline Allocation & & $\mathbf{x}$ & & & & \\
\hline $\begin{array}{l}\text { Assessment of } \\
\text { questionnaires (QoL; } \\
\text { Wexner) }\end{array}$ & $\mathbf{x}$ & & & & & $\mathbf{x}$ \\
\hline Assessment of safety & & $\mathbf{x}$ & $\mathbf{x}$ & $x$ & $\mathbf{x}$ & $\mathbf{x}$ \\
\hline
\end{tabular}

$\mathrm{CCl}$, Comprehensive Complication Index; EoS, end of study; POD, postoperative day; QoL, quality of life. 
representatives are obliged to clarify or explain the queries. At the end of the trial the coordinating investigator will retain the originals of the CRF from the participating sites using paper-based documentation.

\section{Statistical analysis}

All analyses will be exploratory with the main aim being the estimation of standardised effect sizes and CIs as a basis for sample size calculation of a potential subsequent confirmatory (non-inferiority) trial. All patients treated with one of the trial interventions will be considered in the final analysis. The primary analysis strategy will be by modified intention-to-treat, which means that all patients that underwent one of the two interventions under investigation will be assessed in the group that they were randomised to, but patients that did not undergo surgery at all or a completely different procedure will be excluded from the analysis. Per-protocol analysis will be performed as secondary analysis.

The empirical distribution of all endpoints will be calculated, including mean, SD and quartiles in case of continuous variables and scores, and with absolute and relative frequencies in case of categorical data. Then $95 \%$ CIs will be calculated. Descriptive $p$ values of the corresponding statistical tests, $X^{2}$ test for categorical variables and Mann-Whitney $\mathrm{U}$ test for continuous variables, comparing the treatment groups and associated 95\% CIs will be given. Whenever appropriate, statistical graphics will be used to visualise the findings.

The homogeneity of the treatment groups will be described by comparison of the demographic data and the baseline values.

Missing data will be minimised by consequent documentation done in a timely manner and all other reasonable methods. No interpolation of missing data will be performed.

\section{Methods for minimising bias \\ Minimising selection bias}

Patients scheduled for LAR/TME will be consecutively screened for eligibility. The patient flow will be documented and reported with reasons for exclusion at each stage (screening, inclusion, randomisation, analysis) and drop-outs will be explained in a Consolidated Standards of Reporting Trials (CONSORT) flow chart. ${ }^{30}$ If none of the intraoperative exclusion criteria is present, patients will be randomised by means of consecutively numbered and sealed opaque envelopes containing the group allocation. After a telephone call from the operating surgeon to the trial centre, the envelope with the lowest number will be opened by a study nurse and the randomisation result will be announced. A computergenerated randomisation sequence with variable block size will be applied.

\section{Minimising performance and detection bias}

Blinding will be reported for all five trial contributors ${ }^{31}$ : for obvious reasons, treating physicians and patients cannot be blinded to the assigned treatment. Furthermore, all outcomes that could have been assessed by blinded data collectors or outcome assessors were QoL or functional outcomes, that is, patient reported outcomes. Therefore, blinding of data collectors or outcome assessors was not reasonable. Blinded data analysts will perform statistical analyses after closure of the database.

\section{Minimising attrition bias}

Missing data will be minimised by consequent documentation and all other reasonable methods. No interpolation of missing data will be performed. The trial results will be reported according to the recommendations set out in the CONSORT statement. ${ }^{30}$

\section{Minimising reporting bias}

To assure transparent trial conduct and subsequent reporting and to avoid selective reporting, the trial protocol (including full information about its prespecified outcomes and statistical analysis) is published according to the Standard Protocol Items: Recommendations for Interventional Trials statement. ${ }^{32}$

\section{Ethics}

The present trial will be conducted in accordance with the 'Ethical principles for medical research involving human subjects' of the 18th World Medical Association General Assembly in Helsinki (1964), the Declaration of Helsinki in its actual version, ${ }^{33}$ the internationally recognised Good Clinical Practice (ICH-GCP) guidelines, German state and national laws and regulations for data protection, the European General Data Protection Regulation and the German Medical Association's Code of Conduct. The protocol was reviewed and approved by the Ethics Committee of the medical faculty of the University of Heidelberg (S-694/2017).

All patients will be informed by an authorised investigator about the aims of the trial, the possible risks, trial-specific procedures and possible hazards to which the patient will be exposed, and the mechanism of treatment allocation. It will be emphasised that the participation is voluntary and that the patient is allowed to refuse further participation in the trial at any time at the patient's own discretion.

This will not prejudice the patient's subsequent care. Documented informed consent must be obtained from all patients included in the trial before randomisation. The written informed consent form will be signed and personally dated by the patient according to the ICH-GCP guidelines. All patients will get sufficient time to decide on the participation in this trial.

\section{Patient and public involvement}

Patients or the public were not directly involved in the design of the present trial. However, there is abundant literature on the negative impact of ostomies on patients' QoL and bowel function. ${ }^{8435}$ Thus, it may be assumed that avoiding a 'real' ostomy in a substantial proportion of patients by means of the ghost ileostomy, will be of high patient relevance. If novel insights will be available during the conduct of this trial, patients will be promptly informed. Furthermore, it is planned to present and discuss the results of this trial at meetings of patient groups, for example, the German ILCO, 
and to seek patient and public involvement for a potential subsequent confirmatory trial. Furthermore, our group is currently conducting a priority setting partnership, which will bring patients and their relatives together with medical professionals to identify the most important research questions regarding colorectal cancer (www.forschungspartne rschaft.de).

\section{Dissemination}

The final trial results will be published in a peer-reviewed journal. Furthermore, results will be presented at appropriate national and international conferences. Relevant information about the trial and its results will be disseminated to patient groups (eg, the German ILCO) and by social media.

\section{Author affiliations}

${ }^{1}$ Department of General, Visceral and Transplantation Surgery, University of Heidelberg, Heidelberg, Germany

${ }^{2}$ The Study Center of the German Surgical Society (SDGC), University of Heidelberg, Heidelberg, Germany

${ }^{3}$ Surgical Department I, Städtische Kliniken Neuss, Lukaskrankenhaus GmbH, Neuss, Germany

Acknowledgements We thank the members of the data and safety monitoring board: Professor/Dr Peter Sauer (Heidelberg), Professor/Dr Clemens Schafmayer (Kiel) and Professor/Dr Thilo Welsch (Dresden).

Contributors FJH, PP, AM, PC, CD-H and PK developed the trial concept and wrote the protocol and the manuscript of the protocol publication. AU, MS, MWB and MKD helped to develop the trial concept and revised the manuscript critically for important intellectual content. All authors approved the final version for publication agreed to be accountable for all aspects of the work, and ensure that any questions related to the accuracy or integrity of any part of the work will be appropriately investigated and resolved.

Funding This trial was supported by a grant from the Heidelberger Stiftung Chirurgie, a non-profit foundation (grant number: 2017/179).

Disclaimer The funding source of the trial had no role in trial design and will not have a role in data collection, data analysis, interpretation of the trial results or writing of the report.

Competing interests None declared.

Patient consent for publication Not required.

Provenance and peer review Not commissioned; externally peer reviewed.

Supplemental material This content has been supplied by the author(s). It has not been vetted by BMJ Publishing Group Limited (BMJ) and may not have been peer-reviewed. Any opinions or recommendations discussed are solely those of the author(s) and are not endorsed by BMJ. BMJ disclaims all liability and responsibility arising from any reliance placed on the content. Where the content includes any translated material, BMJ does not warrant the accuracy and reliability of the translations (including but not limited to local regulations, clinical guidelines, terminology, drug names and drug dosages), and is not responsible for any error and/or omissions arising from translation and adaptation or otherwise.

Open access This is an open access article distributed in accordance with the Creative Commons Attribution Non Commercial (CC BY-NC 4.0) license, which permits others to distribute, remix, adapt, build upon this work non-commercially, and license their derivative works on different terms, provided the original work is properly cited, appropriate credit is given, any changes made indicated, and the use is non-commercial. See: http://creativecommons.org/licenses/by-nc/4.0/.

\section{ORCID iDs}

Felix J Hüttner http://orcid.org/0000-0002-2299-964X

Pascal Probst http://orcid.org/0000-0002-0895-4015
REFERENCES

1 Ferlay J, Ervik M, Lam F, et al. Global cancer observatory: cancer today. Lyon, France: international agency for research on cancer, 2018. Available: https://gco.iarc.fr/today [Accessed 16 Feb 2020].

2 Rahbari NN, Weitz J, Hohenberger W, et al. Definition and grading of anastomotic leakage following anterior resection of the rectum: a proposal by the International Study group of rectal cancer. Surgery 2010;147:339-51.

3 Leitlinienprogramm Onkologie (Deutsche Krebsgesellschaft DK, AWMF). S3-Leitlinie Kolorektales Karzinom, Langversion 1.1, 2014. Available: http://leitlinienprogramm-onkologie.de/Leitlinien.7.0.html [Accessed 06 Sep 2015].

4 Monson JRT, Weiser MR, Buie WD, et al. Practice parameters for the management of rectal cancer (revised). Dis Colon Rectum 2013;56:535-50.

5 Garg PK, Goel A, Sharma S, et al. Protective diversion stoma in low anterior resection for rectal cancer: a meta-analysis of randomized controlled trials. Visc Med 2019;35:156-60.

6 Chow A, Tilney HS, Paraskeva P, et al. The morbidity surrounding reversal of defunctioning ileostomies: a systematic review of 48 studies including 6,107 cases. Int J Colorectal Dis 2009;24:711-23.

7 Phatak UR, Kao LS, You YN, et al. Impact of ileostomy-related complications on the multidisciplinary treatment of rectal cancer. Ann Surg Oncol 2014;21:507-12.

8 Keane C, Sharma P, Yuan L, et al. Impact of temporary ileostomy on long-term quality of life and bowel function: a systematic review and meta-analysis. ANZ J Surg 2020;90:687-92.

9 Sacchi M, Legge PD, Picozzi P, et al. Virtual ileostomy following tme and primary sphincter-saving reconstruction for rectal cancer. Hepatogastroenterology 2007;54:1676-8.

10 Marrosu A, Serventi F, Pulighe F, et al. The "virtual ileostomy" in elective colorectal surgery: is it useful? Tech Coloproctol 2014;18:319-20.

11 Miccini M, Amore Bonapasta S, Gregori M, et al. Ghost ileostomy: real and potential advantages. Am J Surg 2010;200:e55-7.

12 Mori L, Vita M, Razzetta F, et al. Ghost ileostomy in anterior resection for rectal carcinoma: is it worthwhile? Dis Colon Rectum 2013;56:29-34.

13 Morales-Conde S, Alarcón I, Yang T, et al. A Decalogue to avoid routine ileostomy in selected patients with border line risk to develop anastomotic leakage after minimally invasive Low-Anterior resection: a pilot study. Surg Innov 2020;27:44-53.

14 Palumbo P, Usai S, Pansa A, et al. Anastomotic leakage in rectal surgery: role of the ghost ileostomy. Anticancer Res 2019;39:2975-83.

15 Cerroni M, Cirocchi R, Morelli U, et al. Ghost ileostomy with or without abdominal parietal split. World J Surg Oncol 2011;9:92.

16 Gullà N, Trastulli S, Boselli C, et al. Ghost ileostomy after anterior resection for rectal cancer: a preliminary experience. Langenbecks Arch Surg 2011;396:997-1007.

17 Mari FS, Di Cesare T, Novi L, et al. Does ghost ileostomy have a role in the laparoscopic rectal surgery era? A randomized controlled trial. Surg Endosc 2015;29:2590-7.

18 Baloyiannis I, Perivoliotis K, Diamantis A, et al. Virtual ileostomy in elective colorectal surgery: a systematic review of the literature. Tech Coloproctol 2020;24:23-31.

19 McCulloch P, Altman DG, Campbell WB, et al. No surgical innovation without evaluation: the ideal recommendations. Lancet 2009;374:1105-12.

20 McCulloch P, Cook JA, Altman DG, et al. Ideal framework for surgical innovation 1: the idea and development stages. BMJ 2013;346:f3012.

21 Whitehead AL, Julious SA, Cooper CL, et al. Estimating the sample size for a pilot randomised trial to minimise the overall trial sample size for the external pilot and main trial for a continuous outcome variable. Stat Methods Med Res 2016;25:1057-73.

22 Slankamenac K, Graf R, Barkun J, et al. The comprehensive complication index: a novel continuous scale to measure surgical morbidity. Ann Surg 2013;258:1-7.

23 Dindo D, Demartines N, Clavien P-A. Classification of surgical complications: a new proposal with evaluation in a cohort of 6336 patients and results of a survey. Ann Surg 2004;240:205-13.

24 Kidney Disease: Improving Global Outcomes (KDIGO) Acute Kidney Injury Work Group. KDIGO clinical practice guideline for acute kidney injury. Kidney inter2012:1-138.

25 National Healthcare Safety Network Centers for Disease Control and Prevention. Surgical site infection (SSI) event 2017. Available: http:// www.cdc.gov/nhsn/pdfs/pscmanual/9pscssicurrent.pdf [Accessed 16 Feb 2020].

26 Jorge JM, Wexner SD. Etiology and management of fecal incontinence. Dis Colon Rectum 1993;36:77-97. 
27 Aaronson NK, Ahmedzai S, Bergman B, et al. The European organization for research and treatment of cancer QLQ-C30: a quality-of-life instrument for use in international clinical trials in oncology. J Natl Cancer Inst 1993;85:365-76.

28 Gujral S, Conroy T, Fleissner C, et al. Assessing quality of life in patients with colorectal cancer: an update of the EORTC quality of life questionnaire. Eur J Cancer 2007;43:1564-73.

29 Harris PA, Taylor R, Thielke R, et al. Research electronic data capture (REDCap)--a metadata-driven methodology and workflow process for providing translational research informatics support. J Biomed Inform 2009;42:377-81.

30 Schulz KF, Altman DG, Moher D, et al. CONSORT 2010 statement: updated guidelines for reporting parallel group randomised trials. BMJ 2010;340:c332.
31 Probst P, Zaschke S, Heger P, et al. Evidence-based recommendations for blinding in surgical trials. Langenbecks Arch Surg 2019;404:273-84.

32 Chan A-W, Tetzlaff JM, Altman DG, et al. Spirit 2013 statement: defining standard protocol items for clinical trials. Ann Intern Med 2013;158:200-7.

33 World Medical Association. World Medical association Declaration of Helsinki: ethical principles for medical research involving human subjects. JAMA 2013;310:2191-4.

34 Herrle F, Sandra-Petrescu F, Weiss C, et al. Quality of life and timing of stoma closure in patients with rectal cancer undergoing low anterior resection with diverting stoma: a multicenter longitudinal observational study. Dis Colon Rectum 2016;59:281-90.

35 Schiergens TS, Hoffmann V, Schobel TN, et al. Long-term quality of life of patients with permanent end ileostomy: results of a nationwide cross-sectional survey. Dis Colon Rectum 2017;60:51-60. 